\title{
Cytokine signaling convergence regulates the microglial state transition in Alzheimer's disease
}

\author{
Shun-Fat Lau ${ }^{1,2} \cdot$ Amy K. Y. Fu ${ }^{1,2,3} \cdot$ Nancy Y. Ip $p^{1,2,3}$ \\ Received: 7 January 2021 / Revised: 1 March 2021 / Accepted: 5 March 2021 / Published online: 13 April 2021 \\ (C) The Author(s) 2021
}

\begin{abstract}
Genetic analyses have revealed the pivotal contribution of microglial dysfunctions to the pathogenesis of Alzheimer's disease (AD). Along AD progression, the accumulation of danger-associated molecular patterns (DAMPs) including beta-amyloid and hyperphosphorylated tau continuously stimulates microglia, which results in their chronic activation. Chronically activated microglia secrete excessive pro-inflammatory cytokines, which further regulate microglial responses towards DAMPs. This has spurred longstanding interest in targeting cytokine-induced microglial responses for AD therapeutic development. However, the cytokine-induced microglial state transition is not comprehensively understood. Cytokines are assumed to induce microglial state transition from a resting state to an activated state. However, recent evidence indicate that this microglial state transition involves multiple sequential functional states. Moreover, the mechanisms by which different functional states within the cytokine-induced microglial state transition regulate AD pathology remain unclear. In this review, we summarize how different cytokine signaling pathways, including those of IL-33 (interleukin-33), NLRP3 inflammasome-IL-1 $\beta$, IL-10, and IL-12/IL-23, regulate microglial functions in AD. Furthermore, we discuss how the modulation of these cytokine signaling pathways can result in beneficial outcomes in AD. Finally, we describe a stepwise functional state transition of microglia induced by cytokine signaling that can provide insights into the molecular basis of the beneficial effects of cytokine modulation in $\mathrm{AD}$ and potentially aid therapeutic development.
\end{abstract}

Keywords Interleukin $\cdot$ Chemotaxis $\cdot$ Phagocytosis $\cdot$ Amyloid $\cdot$ Tau

\section{Introduction}

Alzheimer's disease (AD), the most prevalent form of dementia, is characterized by beta-amyloid $(\mathrm{A} \beta)$ deposition and neurofibrillary tangle accumulation [1-3]. The accumulation of these danger-associated molecular patterns (DAMPs) triggers microglial activation in which activated

Nancy Y. Ip

boip@ust.hk

1 Division of Life Science, State Key Laboratory of Molecular Neuroscience, Molecular Neuroscience Center, The Hong Kong University of Science and Technology, Clear Water Bay, Hong Kong, China

2 Hong Kong Center for Neurodegenerative Diseases, Hong Kong, China

3 Guangdong Provincial Key Laboratory of Brain Science, Disease and Drug Development, HKUST Shenzhen Research Institute, Shenzhen-Hong Kong Institute of Brain Science, Shenzhen 518057, Guangdong, China microglia first migrate towards DAMPs and mediate their clearance through phagocytosis $[4,5]$. Activated microglia simultaneously secrete cytokines and immune signaling molecules such as interleukins (ILs) and reactive oxygen species [6, 7]. However, as AD progresses, microglia fail to clear the excess accumulation of DAMPs, resulting in chronic activation and excessive cytokine secretion in the brain.

Therefore, targeting cytokines milieus in the brain has long been a goal of AD research. However, it remains unclear how cytokine signaling should be modulated in $\mathrm{AD}$ to yield beneficial outcomes. One major obstacle is that cytokine-induced microglial responses can elicit both beneficial and detrimental effects. Therefore, it is essential to comprehensively understand cytokine-induced microglial activation in $\mathrm{AD}$.

In this review, we summarize the functional roles of cytokine signaling in mediating the responses of microglia and their communication with other cell types. We also review some of the cytokine signaling pathways that have 
potential therapeutic value for $\mathrm{AD}$, including those of IL-33, NLRP3 inflammasome-IL-1 $\beta$, IL-12/IL-23, and IL-10. Finally, we discuss and propose how cytokines stimulate a stepwise state transition of microglia that leads to the amelioration of $\mathrm{AD}$ pathology.

\section{Neuroinflammation and microglial activation in Alzheimer's disease}

Neuroinflammation is characterized by elevated cytokine levels in the central nervous system microenvironment. In $\mathrm{AD}$, the levels of certain cytokines such as IL- $1 \beta$ and TNF- $\alpha$ (tumor-necrosis factor alpha) increase after the accumulation of $\mathrm{A} \beta$ but before the onset of cognitive impairment, suggesting that neuroinflammation contributes to $\mathrm{AD}$ pathogenesis early on [8-11]. As microglia are the primary immune effectors, their functions are greatly influenced by elevated cytokine levels in AD [12,13]. Accordingly, elevated cytokine levels can regulate various microglial responses and potentially regulate $\mathrm{AD}$ pathogenesis.

Cytokine signaling is essential for the regulation of microglial responses to $A \beta$ pathology. Initial $A \beta$ accumulation triggers microglial activation and cytokine secretion, which in turn stimulate other microglia to react to $A \beta$. As such, several cytokines including IL- $1 \beta$ and IL-33 promote microglial activation and mediate $\mathrm{A} \beta$ clearance $[14,15]$. This suggests that microglial activation, which is induced by transient increases in cytokine levels, can regulate $\mathrm{A} \beta$ clearance and hence incur beneficial effects.

However, microglial activation also incurs adverse effects due to the chronic elevation of cytokine levels. Chronic microglial activation can result in the phagocytosis of non-A $\beta$ materials including synapses and astrocytic processes, which lead to synaptic loss and blood-brain barrier breakdown, respectively [16-18]. Moreover, a recent study demonstrates that increased levels of microglia-derived cytokines including IL- $1 \alpha$ and TNF- $\alpha$ drive the state transition of astrocytes towards a neurotoxic form that mediates neuronal death [19]. Therefore, the duration of cytokineinduced microglial response (i.e., transient vs. chronic) is the distinguishing feature of the functional roles of microglia in $\mathrm{AD}$ pathogenesis.

\section{Cytokine signaling pathways as therapeutic targets for Alzheimer's disease}

Given the role of chronic neuroinflammation in AD pathogenesis, targeting cytokine milieus to modulate microglial functions is an attractive therapeutic approach for AD. Indeed, there have been clinical trials targeting general cytokine milieus using sargramostim [20], a small molecule
RAGE inhibitor [21], NSAIDs [22, 23], and etanercept [24] in patients with $\mathrm{AD}$ or mild cognitive impairment [25]. However, most of them were aborted owing to a lack of efficacy, suggesting that simply inhibiting the secretion of pro-inflammatory cytokines cannot effectively restore brain homeostasis or improve cognitive performance in patients with AD.

These failures also underscore our incomplete understanding of the roles and immunomodulatory functions of specific cytokine signaling pathways in $\mathrm{AD}$. This knowledge gap is partly due to the lack of unbiased, comprehensive profiling of different cytokines along with $\mathrm{AD}$ progression. While previous studies using candidate approaches show that dysregulated levels of several specific cytokines including IL-33, NLRP3 inflammasome, IL-12/IL-23, and IL-10 are associated with AD pathogenesis [15, 26-30], modulating these cytokine signaling pathways in mouse models of amyloidosis and hyperphosphorylated tau results in beneficial outcomes. Accordingly, below we review the beneficial effects of modulating these cytokine signaling pathways in AD.

\section{IL-33 replenishment ameliorates Alzheimer's disease pathology by promoting a PU.1-dependent microglial state transition}

IL-33 is an IL-1 family member that functions as an alarmin to trigger an immune response to cellular damage or injury [31, 32]. Interestingly, IL-33 is most abundant in the brain and lungs [33]. In the brain, IL-33 is enriched within the nuclei of oligodendrocytes but is also found in astrocytes and neurons [33, 34]. Nuclear IL-33 is inactive and interacts with histones [35]. Upon central nervous system injury, IL-33 is rapidly released by necrotic or apoptotic cells and simulates microglial recruitment [31, 33, 36, 37].

Genetic studies demonstrate that AD pathogenesis is associated with single nucleotide polymorphisms of IL-33 and its receptor ST2 [38, 39]. Specifically, patients with AD and mild cognitive impairment exhibit reduced gene expression of IL33 and increased serum levels of soluble ST2 (the decoy receptor of IL-33), respectively [15, 39]. This suggests that IL-33/ST2 signaling is impaired in AD. Concordantly, IL-33 deficiency can drive tau pathology and neuronal loss in aged mice [40]. In addition, in the APP/PS1 mouse model of amyloid deposition, IL-33 administration can ameliorate synaptic impairment, improve cognitive performance, and reduce levels of $\mathrm{A} \beta$ and pro-inflammatory cytokines (e.g., IL-1 $\beta$, IL-6, and NLRP3 inflammasome) [15]. These findings collectively show that restoring IL-33/ST2 signaling can alleviate $\mathrm{AD}$ pathology.

Microglia mediate these beneficial outcomes of replenishing IL-33 in AD. IL-33 administration triggers the state transition of microglia and induces a subpopulation of 
IL-33-responsive microglia that have a distinct transcriptomic signature characterized by increased expression of major histocompatibility complex class II genes and homeostatic signature genes as well as enhanced $\mathrm{A} \beta$-phagocytic and clearance capacity [26]. Furthermore, epigenetic analyses show that IL-33 can remodel the chromatin accessibility and PU.1-binding landscapes in microglia, resulting in the induction of IL-33-responsive microglia. These lines of evidence delineate the molecular mechanisms of the IL-33-induced microglial state transition and its beneficial outcomes. However, given that PU.1 functions as a lineagedependent transcription factor and is unlikely to be direct downstream of IL-33/ST2 signaling, further investigation is required to understand how PU.1 cooperates with transcription factors downstream of IL-33 to mediate the induction of IL-33-responsive microglia. Nonetheless, these findings show that restoring IL-33/ST2 signaling in AD promotes the clearance activity of microglia via PU.1-dependent transcriptional control, which yields beneficial outcomes.

\section{NLRP3 inflammasome activation exacerbates $A \beta$ and tau pathology}

NLRP3 inflammasome is a multiprotein complex that consists of the sensor NLRP3 (NACHT-, LRR-, and pyrin [PYD] domain-containing protein 3), adaptor ASC, and effector pro-caspase-1 [41]. NLRP3 inflammasome acts a cytosolic pattern-recognition receptor that mediates the sensing of DAMPs such as $A \beta[41,42]$. Upon activation by A $\beta$, NLRP3, ASC, and pro-caspase- 1 self-assemble, which results in the cleavage of pro-caspase- 1 into proteolytically active caspase-1 [43]. In turn, this active caspase-1 can cleave pro-IL- $1 \beta$ and pro-IL-18 to generate the mature forms of IL-1 $\beta$ and IL-18, respectively. Meanwhile, inhibiting caspase- 1 activity attenuates $A \beta$-induced IL- $1 \beta$ production in microglia [43]. These findings show that NLRP3 plays an important role in the regulation of IL- $1 \beta$ production by microglia in $\mathrm{AD}$.

Besides controlling the release of cytokines, NLRP3 inflammasome contributes to other aspects of AD pathogenesis. First, NLRP3 contributes to amyloid pathology by regulating the phagocytic capacity of microglia in $\mathrm{AD}$. Genetic ablation of NLRP3 in APP/PS1 mice enhances the $\mathrm{A} \beta$-phagocytic capacity of microglia, which reduces the level of $A \beta$ in the brain [27]. This coincides with the induction of anti-inflammatory microglial phenotypes characterized by increased gene expression of Arg1, Fizz1, Il4, and Nos2. However, it remains unclear how these anti-inflammatory microglia contribute to the enhanced phagocytic capacity of microglia. Second, activated microglia can secrete ASC, a component of NLRP3 inflammasome, to bind and cross-seed $A \beta$ peptides to form $A \beta$ plaques [44]. Once released into the extracellular space, ASC can bind $\mathrm{A} \beta$ peptides to form the core of an $\mathrm{A} \beta$ deposit and facilitate further aggregation of $A \beta$. This effect of ASC-mediated A $\beta$ seeding also contributes to the accumulation of A $\beta$ deposition in AD. Finally, NLRP3 inflammasome activation also regulates tau pathology in $\mathrm{AD}$ [45]. The reduction of NLRP3 activation in a mouse model of tau pathology is associated with reduced activity of CaMKII $\alpha$ and GSK3 $\beta$ (kinases that phosphorylate tau) and increased activity of PP2A (a phosphatase that dephosphorylates tau) [46]. Thus, reducing NLRP3 inflammasome activation contributes to the overall reduction in tau pathology and improves cognitive performance in mice.

These findings collectively demonstrate that the action of NLRP3 inflammasome in AD contributes to amyloid and tau pathology through the modulation of the clearance activities of microglia, $A \beta$ seeding, and kinase activities.

\section{IL-12/IL-23 inhibition alleviates $A \beta$ pathology in a sex-specific manner}

IL-12 and IL-23 structurally share the same IL-12p40 protein subunit, which links to the IL-12p35 and IL-23p19 subunits, respectively $[47,48]$. While IL-12/IL-23 dysregulation has long been considered to drive the pathogeneses of several autoimmune diseases such as experimental autoimmune encephalomyelitis and arthritis [47, 49], recent evidence shows that dysregulated IL-12/IL-23 signaling can also contribute to AD. In patients with AD, IL-12p40 is elevated in the cerebrospinal fluid [28], which is associated with impaired cognitive performance. Thus, aberrant IL-12/IL-23 signaling contributes to AD pathogenesis, suggesting that its inhibition might produce beneficial outcomes in AD. Indeed, inhibiting IL-12/IL-23 signaling either by the administration of an IL-12p40-neutralizing antibody or genetic ablation of IL-12/IL-23 subunits ameliorates $A \beta$ pathology and cognitive impairment in APP/PS1 mice [28]. Interestingly, a recent study also shows that the beneficial effect of inhibiting IL-12/IL-23 exhibits a sex-specific bias in APP23 transgenic mice [50]. While genetic ablation of IL-12p40 reduces the total A $\beta$ level in male APP23 mice, it only reduces the soluble $\mathrm{A} \beta_{1-40}$ level in female APP23 mice. However, it remains unclear how sex contributes to this sex-specific beneficial effect of IL-12/IL-23 inhibition in AD. More importantly, the IL-12/IL-23 receptor gene $1112 r b 1$ is predominately expressed in oligodendrocytes and other non-myeloid cells $[28,51]$. Therefore, it is unclear how inhibiting IL-12 and/ or IL-23 signaling elicits beneficial effects through the regulation of the cell-state transition of non-myeloid cells in AD. Nonetheless, these findings collectively demonstrate that inhibiting IL-12/IL-23 signaling in AD can alleviate amyloid pathology in a sex-specific manner. 


\section{IL-10 inhibition alleviates A $\beta$ pathology through the regulation of microglial activation status}

IL-10 is known for its role in the suppression of immune responses in peripheral immunity and is, therefore, considered an anti-inflammatory cytokine. IL-10 suppresses the secretion of pro-inflammatory cytokines including TNF- $\alpha$, inflammasome activation, and the induction of major histocompatibility complex class II and co-stimulatory molecules in macrophages and dendritic cells [52-54]. The immunosuppressive effects of IL-10 are partly mediated through the activation of STAT3-dependent transcriptional control by its receptor, IL-10 receptor [55]. Thus, IL-10 plays a key role in restraining aberrant immune activation and dysregulated signaling associated with various immune-related diseases including AD.

Genetic analyses of patients with AD have identified that IL-10 promoter polymorphism is associated with AD risk in Chinese and Italian cohorts, suggesting that dysregulated IL-10 signaling potentially contributes to $\mathrm{AD}[56$, 57]. Building on this finding, one study demonstrates that genetic ablation of IL-10 in APP/PS1 mice ameliorates A $\beta$ pathology by promoting microglial activation [29]. Furthermore, subsequent transcriptome analysis showed that IL-10 ablation suppresses the gene signature of disease-associated microglia (i.e., Apoe, Clec7a, Itgax, and Trem2), probably due to the reduced $A \beta$ level in the brain. On the other hand, IL-10 overexpression mediated by adeno-associated virus exacerbates $A \beta$ pathology and cognitive impairment in TgCRND8 mice, another mouse model that overexpresses mutant human APP [30]. IL-10 overexpression stimulates ApoE (apolipoprotein $\mathrm{E}$ ) production and aggregation with $A \beta$, thus inhibiting microglial $A \beta$ phagocytosis. These findings collectively demonstrate that inhibiting IL-10 signaling ameliorates $\mathrm{AD}$ pathology through the activation of microglia.

\section{Cytokine signaling convergence and competition in Alzheimer's disease}

One intriguing observation stemming from the abovementioned findings is that all modulation of cytokine signaling - activation of IL-33, or inhibition of NLRP3 inflammasome-IL-1 or IL-10-promotes microglial activation and ameliorates $\mathrm{A} \beta$ pathology in AD (Table 1). While activating IL-33 signaling promotes microglial activation, why does inhibiting cytokine signaling elicit a similar effect? To answer this, we must first understand the interplay among cytokine signaling pathways within microglia. Here, we use IL-33, NLRP3 inflammasome-IL-1 $\beta$, and IL-10 signaling to illustrate such signal interplay in microglia; we excluded IL-12/IL-23 signaling because microglia are not the primary target cells that respond to the modulation of these two cytokines.

All three cytokine signaling pathways-IL-33, NLRP3 inflammasome-IL-1 $\beta$, and IL-10—converge during signal transduction steps. For example, IL-33 triggers its downstream signaling by binding to ST2 and IL-1RAP heterodimers (Fig. 1a). This dimerizes the cytosolic adaptor protein MyD88 along with IRAK (IL-1R-associated kinase) to activate transcriptional control dependent on both AP-1 (activator protein 1) and NF- $\kappa \mathrm{B}$, which closely resembles the classical IL-1 signaling cascade [32]. Similar to IL-33, IL-1 signaling is one of the downstream signaling pathways of NLRP3 inflammasome activation. This is because activated NLRP3 inflammasome produces mature IL-1 $\beta$ for secretion. Once secreted, IL-1 $\beta$ acts on microglia via IL-1R to trigger the IL-1 signaling cascade (Fig. 1b) [58]. While IL-10 activates distinct sets of downstream signaling pathways (i.e., STAT3 via JAK1) [59], IL-10 activation can induce the ubiquitination of IRAK and TRAF, which are core signal transducers of the IL-1 signaling cascade, to limit IL-1-dependent responses (Fig. 1c) [60]. Hence, these findings collectively illustrate how the cytokine signaling pathways interact and converge at the signal transduction stage within microglia.

Because of this signaling convergence, the simultaneous activation of various cytokine signaling pathways due to the chronically elevated cytokine levels in AD triggers competition for the same set of intracellular signal transducers, which leads to mutual inhibition (Fig. 1d). This phenomenon is prominent in the peripheral immune response. For example, given that IL-33 and IL-1 employ the same downstream signaling cascade, the activation of ST2 signaling can inhibit IL-1R signaling or vice versa. Indeed, compared to control mice, myeloid cells from ST2-deficient mice produce significantly more pro-inflammatory cytokines when systemically challenged with IL-1 [61]. Besides myeloid cells, natural killer cells from ST2-deficient mice also exhibit enhanced production of pro-inflammatory cytokines such as IFN- $\gamma$ [62]. Therefore, we hypothesize that suppressing pro-inflammatory cytokine signaling (e.g., that of NLRP3 inflammasome-IL-1 $\beta$ ) relieves its inhibition of the signaling pathways of other cytokines (e.g., IL-33) and hence promotes beneficial microglial activation in AD (Fig. 1d).

Besides the abovementioned signaling competition, there are other possible negative feedback mechanisms within the cytokine signaling cascade. At the signal transduction level, IRAK-M, an inactive kinase downstream of Toll-like receptor, can inhibit the receptor signaling in macrophages by dissociating the IRAK signaling complex [63]. Also, SIGIRR, an IL-1R-related surface receptor, can inhibit IL-1 signaling by sequestering downstream signaling molecules [64]. Furthermore, at the transcriptional level, exaggerated cytokine response 


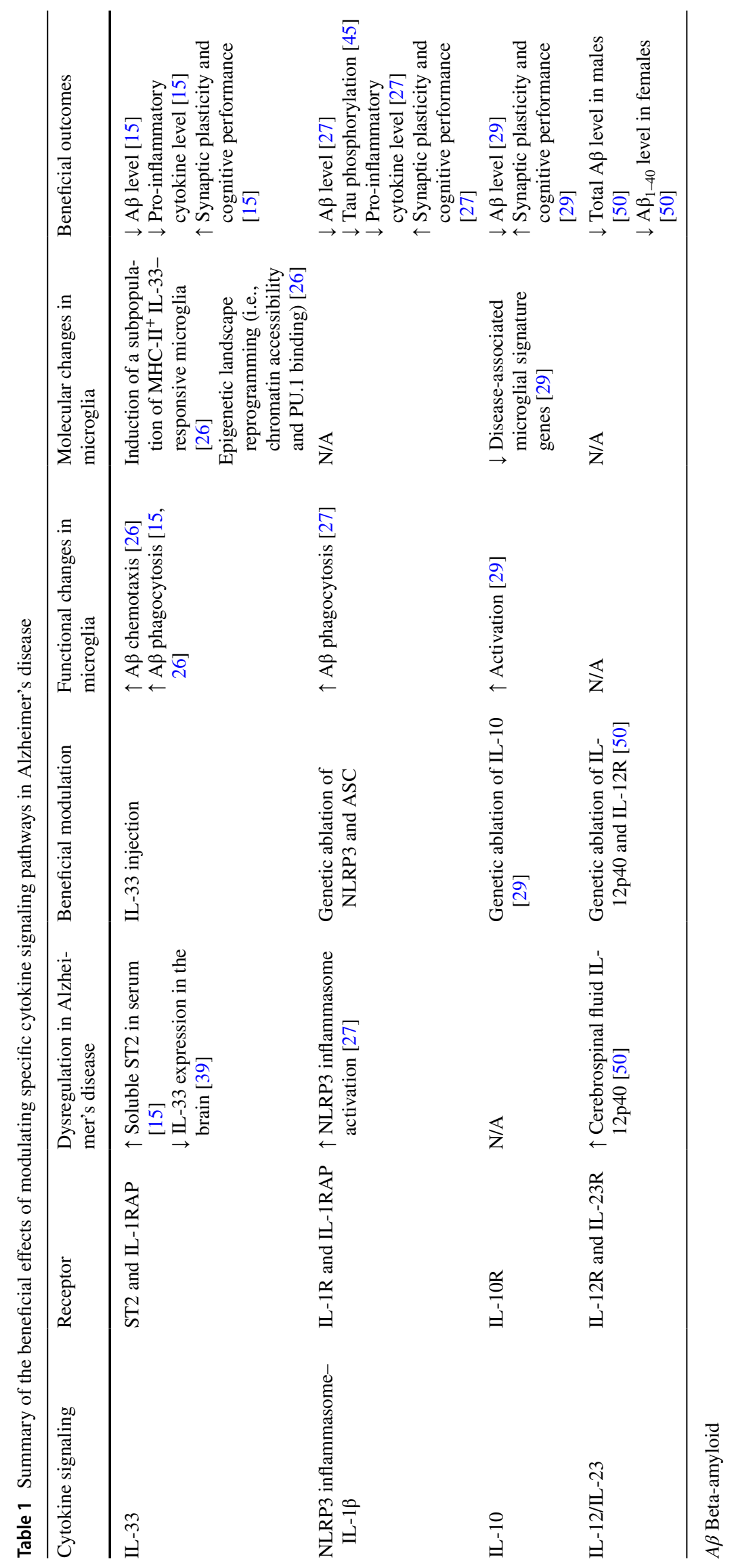


Fig. 1 Interplay among cytokine signaling pathways within microglia. a-c Diagrams illustrating the signaling pathways downstream of IL-33 (a), NLRP3 inflammasome-IL-1 $\beta$ (b), and IL-10 (c). d Diagram illustrating the inhibitory effects of the simultaneous activation of multiple cytokine signaling pathways on neighboring pathways. $A \beta$, beta-amyloid
A

IL-33

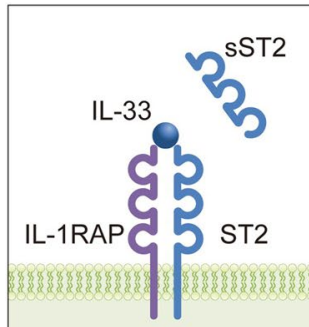

MyD88 \& IRAK

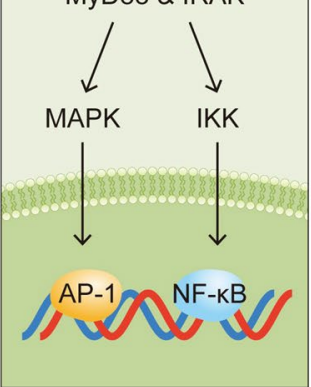

B NLRP3 inflammasome-IL-1 $\beta$

C IL-10
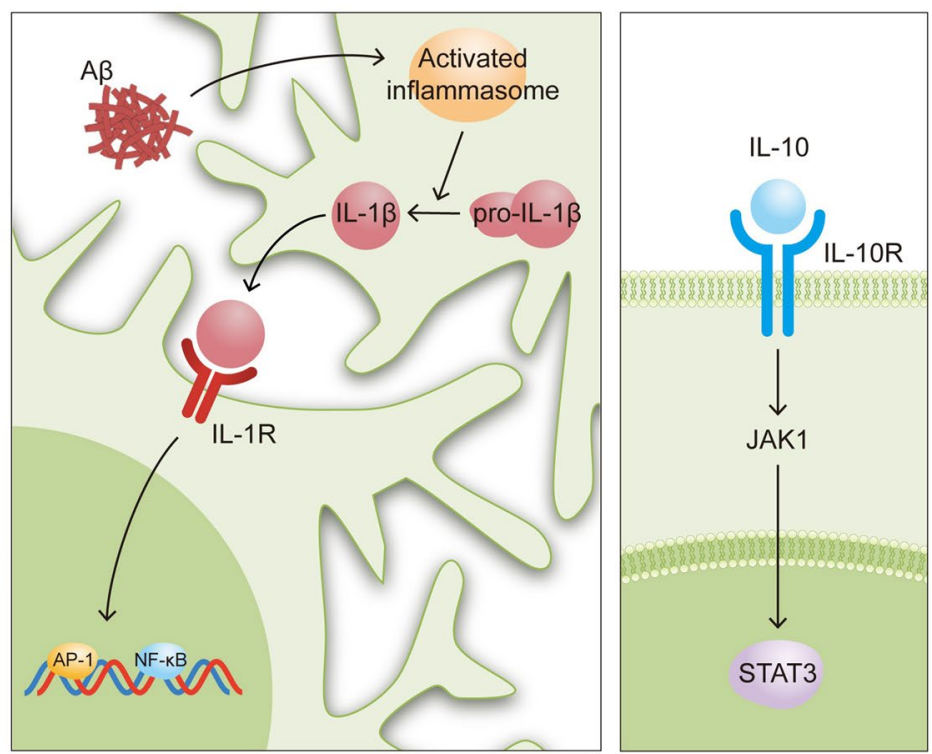

D

Signal integration among IL-33, IL-1 $\beta$, and IL-10 signaling

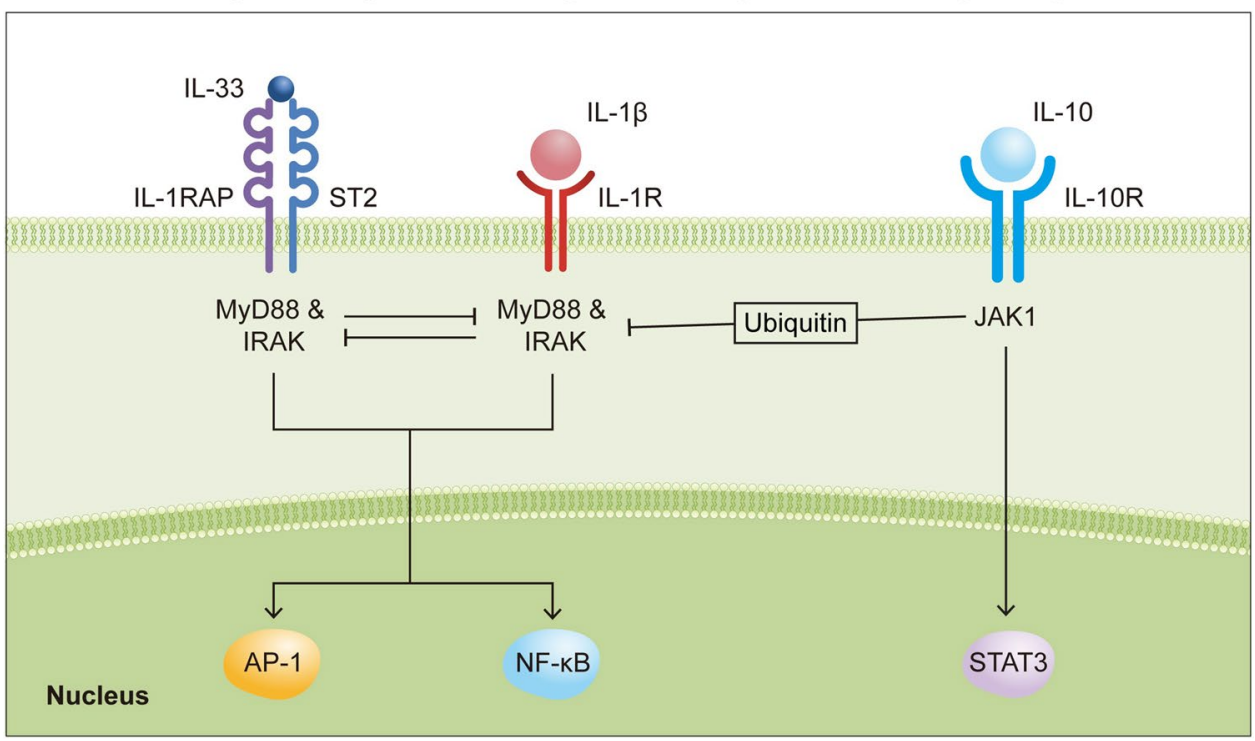

can be limited by stimulating the gene expression of decoy receptors and receptor antagonists upon cytokine activation. For example, IL-33 stimulates the expression of soluble ST2 (an IL-33 decoy receptor), and IL-1RA (an IL-1 receptor antagonist) to limit further cytokine activation $[65,66]$. Therefore, when developing therapeutic strategies for $\mathrm{AD}$ based on the modulation of cytokine signaling, whether feedback mechanisms in cytokine signaling affect the expected beneficial outcome (e.g., microglial activation) must be considered. Accordingly, we propose targeting the cytokine inhibitory signal-for example by cytokine competition or decoy receptors of cytokines-as an alternative approach to promote the beneficial functions of microglia in AD.

\section{Stepwise microglial state transition upon cytokine activation in Alzheimer's disease}

The association between microglial activation and $\mathrm{AD}$ has been reported in patients for decades. However, its molecular basis was only recently elucidated through the advancement of single-cell transcriptome analysis. Studies profiling the microglial transcriptome at the single-cell level in various mouse models of amyloid deposition have identified the induction of microglial subtypes including disease-associated microglia and activated response microglia $[67,68]$. These microglial subtypes share similar transcriptome profiles-for example, increased expression 
of AD risk genes including APOE and TREM2 as well as genes associated with lysosomal pathways-and are colocalized with $\mathrm{A} \beta$ plaques. These findings suggest that as $\mathrm{AD}$ progresses, microglia gradually adopt an activated state in response to the deterioration of the central nervous system microenvironment.

Interestingly, the activation status of microglia in $\mathrm{AD}$ is highly dynamic and reversible. For example, upon cytokine activation in $\mathrm{AD}$, alternative activation states of microglia can be induced, such as anti-inflammatory phenotypes by NLRP3 inhibition or IL-33-responsive microglia by IL-33 activation [26, 27]. However, it remains unclear how these activated microglia differentiate from their precursor cells. Given that microglia robustly react to stimuli (i.e., within 1-h post-activation) [69,70], comprehensive time-course profiling of the microglial transcriptome and functional characterization are essential to understand the molecular mechanisms underlying cytokine-induced microglial activation and its beneficial effects in AD.

Despite these unknowns, by summarizing the microglial phenotypes observed from the abovementioned studies, we propose a stepwise state transition model to delineate the beneficial cytokine-induced response of microglia in AD. Upon cytokine activation, microglia transcriptomically and functionally transition from a homeostatic state to a chemotactic state and finally to a phagocytic state in AD (Fig. 2). Here, we further discuss the two key states of the stepwise state transition of microglia-the chemotactic and phagocytic states-as well as their molecular regulators.

\section{Chemotactic state}

Chemotaxis is the directional migration of a cell towards a particular ligand. To achieve this, microglia express specific surface receptors that sense their corresponding ligands. Given that transcriptomic profile reprogramming drives the functional state of microglia, the enhanced $\mathrm{A} \beta$ chemotaxis upon cytokine activation is likely due to increased expression of surface receptors that mediate the sensing of $A \beta$ and its associated proteins such as ApoE [71, 72]. Several cell-surface receptors including TLR2 and TREM2 regulate the interactions of microglia with $\mathrm{A} \beta$ and ApoE [73-76]. Genetic ablation, particularly that of TREM2, impairs the recruitment of microglia towards $\mathrm{A} \beta$ as well as ApoEdependent transcriptomic reprogramming of microglia (Fig. 2). Moreover, unpublished data from our laboratory indicate that IL-33 induces a repertoire of A $\beta$-sensing receptors shortly after administration in APP/PS1 mice. These findings collectively suggest that cytokine signaling can induce the expression of $A \beta$-sensing receptors and regulate microglial chemotaxis towards $\mathrm{A} \beta$.

\section{Phagocytic state}

Once microglia reach $A \beta$ plaques, they mediate $A \beta$ clearance via phagocytosis (Fig. 2). Similar to chemotaxis, the phagocytic capacity of microglia is also regulated by their transcriptomic signature, which determines the expression of phagocytic receptors, trafficking molecules, and proteindegrading enzymes. For example, $C D 33$, an AD risk gene that encodes a sialic acid-binding immunoglobulin-like lectin, can inhibit the microglial uptake of neurotoxic $A \beta_{42}$ [77]. Once $A \beta$ is internalized, phagocytic vesicles containing $A \beta$ enter lysosomal pathways, which leads to $A \beta$ degradation. Interestingly, many AD risk genes such as BIN1, PICALM, and SORL1 are important regulators for the trafficking of phagocytic vesicles [78]. While these findings show that a gene signature related to phagolysosomal pathways is crucial for the regulation of microglial phagocytosis, it remains largely unclear if cytokines control the phagocytic capacity of microglia by modulating the expression of such pathways in $\mathrm{AD}$.

Therefore, additional detailed investigations are required to delineate how the gene signature of each microglial state contributes to the corresponding functions of microglia in AD. As such, we propose a stepwise state transition model to demonstrate the cytokine-stimulated microglial response
Fig. 2 Proposed model of the stepwise microglial state transition stimulated by cytokine signaling. Colored bars indicate the expression level of each microglial transcriptomic signature during cytokine-induced state transition, with thicker bars indicating higher expression

\section{Transcriptome}

$\begin{array}{cl} & \text { Candidate genes } \\ \text { Homeostatic } & \text { Fcrls, Hexb, P2ry12, \& Tmem119 } \\ \text { Chemotactic } & \text { TIr2 \& Trem2 } \\ \text { Phagocytic } & \text { Bin1, Cd33, Picalm, \& Sorl1 }\end{array}$

Function

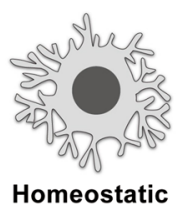

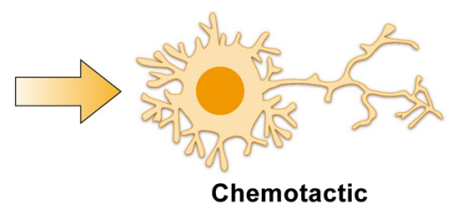

Chemotactic

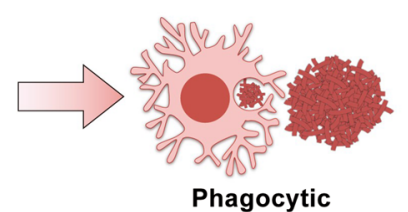


in $\mathrm{AD}$ in which microglia transition from a homeostatic to a chemotactic state and finally to a phagocytic state.

\section{Concluding remarks}

Impaired $\mathrm{A} \beta$ clearance is strongly associated with the pathogenesis of late-onset $\mathrm{AD}$, which accounts for over $95 \%$ of $\mathrm{AD}$ cases. Therefore, enhancing microglial $\mathrm{A} \beta$ clearance is a promising therapeutic approach for $\mathrm{AD}$. While targeting the cytokine signaling pathways discussed herein has great therapeutic potential, these pathways also trigger strong immune responses in the peripheral system, which limits their translational potential. Therefore, it is essential to understand the molecular and cellular bases of such beneficial outcomesespecially how these pathways promote the state transition and clearance activity of microglia. Indeed, as illustrated above, the microglial state transition is controlled at multiple levels by extracellular signals, signal transducers, transcription factors, and transcriptomes, suggesting that there are multiple targets for manipulating microglial states at each level. Accordingly, in-depth investigations of how the interplay among signaling transducers and transcription factors controls the microglial state transition in $\mathrm{AD}$ are required to identify precise molecular targets for enhancing the microglial state transition and restoring brain homeostasis in AD.

Acknowledgements We thank Ka Chun Lok for the excellent illustrations as well as all other members of the Ip Laboratory for many helpful discussions. This study was supported in part by the Research Grants Council of Hong Kong [the Collaborative Research Fund (C6027-19GF), the Theme-Based Research Scheme (T13-607/12R), and the General Research Fund (HKUST16149616, HKUST16103017, HKUST16100418, and HKUST16102019)], the National Key R\&D Program of China Grants (2017YFE0190000 and 2018YFE0203600), the Areas of Excellence Scheme of the University Grants Committee (AoE/M-604/16), the Innovation and Technology Commission Grant (ITCPD/17-9), the Guangdong Provincial Key S\&T Program Grant (2018B030336001), and Shenzhen Knowledge Innovation Program Grants (JCYJ20180507183642005 and JCYJ20170413173717055). S.-F.L. is a recipient of the Hong Kong PhD Fellowship Award.

Open Access This article is licensed under a Creative Commons Attribution 4.0 International License, which permits use, sharing, adaptation, distribution and reproduction in any medium or format, as long as you give appropriate credit to the original author(s) and the source, provide a link to the Creative Commons licence, and indicate if changes were made. The images or other third party material in this article are included in the article's Creative Commons licence, unless indicated otherwise in a credit line to the material. If material is not included in the article's Creative Commons licence and your intended use is not permitted by statutory regulation or exceeds the permitted use, you will need to obtain permission directly from the copyright holder. To view a copy of this licence, visit http://creativecommons.org/licenses/by/4.0/.

\section{References}

1. Reitz C, Brayne C, Mayeux R (2011) Epidemiology of Alzheimer disease. Nat Rev Neurol 7:137-152

2. Selkoe DJ, Hardy J (2016) The amyloid hypothesis of Alzheimer's disease at 25 years. EMBO Mol Med 8:595-608

3. Herrup K (2015) The case for rejecting the amyloid cascade hypothesis. Nat Neurosci 18:794-799

4. Heneka MT, Golenbock DT, Latz E (2015) Innate immunity in Alzheimer's disease. Nat Immunol 16:229-236

5. Heneka MT et al (2015) Neuroinflammation in Alzheimer's disease. Lancet Neurol 14:388-405

6. Perry VH, Nicoll JAR, Holmes C (2010) Microglia in neurodegenerative disease. Nat Rev Neurol 6:193-201

7. Ransohoff RM, Perry VH (2009) Microglial physiology: unique stimuli, specialized responses. Annu Rev Immunol 27:119-145

8. Wang WY, Tan MS, Yu JT, Tan L (2015) Role of pro-inflammatory cytokines released from microglia in Alzheimer's disease. Ann Transl Med 3:136

9. Zheng C, Zhou XW, Wang JZ (2016) The dual roles of cytokines in Alzheimer's disease: Update on interleukins, TNF- $\alpha$, TGF- $\beta$ and IFN- $\gamma$. Transl Neurodegener 5:1-15

10. Holmes $C$ et al (2009) Systemic inflammation and disease progression in alzheimer disease. Neurology 73:768-774

11. Motta M, Imbesi R, Di Rosa M, Stivala F, Malaguarnera L (2007) Altered plasma cytokine levels in Alzheimer's disease: correlation with the disease progression. Immunol Lett $114: 46-51$

12. Solfrizzi V et al (2006) Circulating biomarkers of cognitive decline and dementia. Clin Chim Acta 364:91-112

13. Leung $\mathrm{R}$ et al (2013) Inflammatory proteins in plasma are associated with severity of Alzheimer's disease. PLoS One 8:e64971

14. Shaftel SS et al (2007) Sustained hippocampal IL-1 $\beta$ overexpression mediates chronic neuroinflammation and ameliorates Alzheimer plaque pathology. J Clin Invest 117:1595-1604

15. Fu AKY et al (2016) IL-33 ameliorates Alzheimer's diseaselike pathology and cognitive decline. Proc Natl Acad Sci USA 113:E2705-E2713

16. Haruwaka K et al (2019) Dual microglia effects on blood brain barrier permeability induced by systemic inflammation. Nat Commun 10:1-17

17. Hong $\mathrm{S}$ et al (2016) Complement and microglia mediate early synapse loss in Alzheimer mouse models. Science (-80) 352:712-716

18. Schafer DP, Stevens B (2015) Microglia function in central nervous system development and plasticity. Cold Spring Harb Perspect Biol 7:a020545

19. Liddelow SA et al (2017) Neurotoxic reactive astrocytes are induced by activated microglia. Nature 541:481-487

20. Grant SM, Heel RC (1992) Recombinant granulocyte-macrophage colony-stimulating factor (rGM-CSF): a review of its pharmacological properties and prospective role in the management of myelosuppression. Drugs 43:516-560

21. Deane R et al (2012) A multimodal RAGE-specific inhibitor reduces amyloid $\beta$-mediated brain disorder in a mouse model of Alzheimer disease. J Clin Invest 122:1377-1392

22. Stewart WF, Kawas C, Corrada M, Metter EJ (1997) Risk of Alzheimer's disease and duration of NSAID use. Neurology 48:626-632

23. McGeer PL, Rogers J, McGeer EG (2016) Inflammation, antiinflammatory agents, and Alzheimer's disease: the last 22 years. J Alzheimer's Dis 54:853-857

24. Tufan AN, Tufan F (2015) Etanercept in Alzheimer disease: a randomized, placebo-controlled, double-blind, phase 2 trial. Neurology 85:2083-2084 
25. Fu WY, Wang X, Ip NY (2018) Targeting neuroinflammation as a therapeutic strategy for Alzheimer's disease: mechanisms, drug candidates, and new opportunities. ACS Chem Neurosci. https:// doi.org/10.1021/acschemneuro.8b00402

26. Lau SF et al (2020) IL-33-PU.1 transcriptome reprogramming drives functional state transition and clearance activity of microglia in Alzheimer's disease. Cell Rep 31:107530

27. Heneka MT et al (2013) NLRP3 is activated in Alzheimer's disease and contributes to pathology in APP/PS1 mice. Nature 493:674-678

28. Vom Berg $\mathbf{J}$ et al (2012) Inhibition of IL-12/IL-23 signaling reduces Alzheimer's diseasea-like pathology and cognitive decline. Nat Med 18:1812-1819

29. Guillot-Sestier MV et al (2015) I110 deficiency rebalances innate immunity to mitigate Alzheimer-like pathology. Neuron 85:534-548

30. Chakrabarty P et al (2015) IL-10 alters Immunoproteostasis in APP mice, increasing plaque burden and worsening cognitive behavior. Neuron 85:519-533

31. Liew FY, Girard JP, Turnquist HR (2016) Interleukin-33 in health and disease. Nat Rev Immunol 16:676-689

32. Carriere V et al (2007) IL-33, the IL-1-like cytokine ligand for ST2 receptor, is a chromatin-associated nuclear factor in vivo. Proc Natl Acad Sci USA 104:282-287

33. Gadani SP, Walsh JT, Smirnov I, Zheng J, Kipnis J (2015) The glia-derived alarmin IL-33 orchestrates the immune response and promotes recovery following CNS injury. Neuron 85:703-709

34. Vainchtein ID et al (2018) Astrocyte-derived interleukin-33 promotes microglial synapse engulfment and neural circuit development. Science 359:1269-1273

35. Travers J et al (2018) Chromatin regulates IL-33 release and extracellular cytokine activity. Nat Commun 9:1-15

36. Cayrol C, Girard JP (2014) IL-33: An alarmin cytokine with crucial roles in innate immunity, inflammation and allergy. Curr Opin Immunol 31:31-37

37. Kakkar R, Lee RT (2008) The IL-33/ST2 pathway: Therapeutic target and novel biomarker. Nat Rev Drug Discov 7:827-840

38. Yu JT et al (2012) Implication of IL-33 gene polymorphism in Chinese patients with Alzheimer's disease. Neurobiol Aging 33(1014):e11-1014.e14

39. Chapuis J et al (2009) Transcriptomic and genetic studies identify IL-33 as a candidate gene for Alzheimer's disease. Mol Psychiatry 14:1004-1016

40. Carlock $\mathrm{C}$ et al (2017) Interleukin33 deficiency causes tau abnormality and neurodegeneration with Alzheimer-like symptoms in aged mice. Transl Psychiatry 7:e1164-e1164

41. Swanson KV, Deng M, Ting JPY (2019) The NLRP3 inflammasome: molecular activation and regulation to therapeutics. Nat Rev Immunol 19:477-489

42. Heneka MT (2017) Inflammasome activation and innate immunity in Alzheimer's disease. Brain Pathol 27:220-222

43. Halle A et al (2008) The NALP3 inflammasome is involved in the innate immune response to amyloid- $\beta$. Nat Immunol 9:857-865

44. Venegas $C$ et al (2017) Microglia-derived ASC specks crossseed amyloid- $\beta$ in Alzheimer's disease. Nature 552:355-361

45. Ising $C$ et al (2019) NLRP3 inflammasome activation drives tau pathology. Nature 575:669-673

46. Iqbal $\mathrm{K}$ et al (2005) Tau pathology in Alzheimer disease and other tauopathies. Biochim Biophys Acta Mol Basis Dis 1739:198-210

47. Khader SA, Thirunavukkarasu S (2019) The tale of IL-12 and IL-23: a paradigm shift. J Immunol 202:629-630

48. Teng MWL et al (2015) IL-12 and IL-23 cytokines: from discovery to targeted therapies for immune-mediated inflammatory diseases. Nat Med 21:719-729

49. Croxford AL, Kulig P, Becher B (2014) IL-12-and IL-23 in health and disease. Cytokine growth factor rev 25:415-421
50. Eede $\mathrm{P}$ et al (2020) Interleukin-12/23 deficiency differentially affects pathology in male and female Alzheimer's disease-like mice. EMBO Rep. 21:e48530

51. Zhang Y et al (2014) An RNA-sequencing transcriptome and splicing database of glia, neurons, and vascular cells of the cerebral cortex. J Neurosci 34:11929-11947

52. Couper KN, Blount DG, Riley EM (2008) IL-10: the master regulator of immunity to infection. J Immunol 180:5771-5777

53. Iyer SS, Cheng G (2012) Role of interleukin 10 transcriptional regulation in inflammation and autoimmune disease. Crit Rev Immunol 32:23-63

54. Ipadc WKE, Hoshi N, Shouval DS, Snapper S, Medzhitov R (2017) Anti-inflammatory effect of IL-10 mediated by metabolic reprogramming of macrophages. Science (80-). 356:513-519

55. Hutchins AP, Diez D, Miranda-Saavedra D (2013) The IL-10/ STAT3-mediated anti-inflammatory response: Recent developments and future challenges. Brief Funct Genomics 12:489-498

56. Lio D et al (2003) Interleukin-10 promoter polymorphism in sporadic Alzheimer's disease. Genes Immun 4:234-238

57. Ma SL, Tang NLS, Lam LCW, Chiu HFK (2005) The association between promoter polymorphism of the interleukin-10 gene and Alzheimer's disease. Neurobiol Aging 26:1005-1010

58. He Y, Hara H, Núñez G (2016) Mechanism and regulation of NLRP3 inflammasome activation. Trends Biochem Sci 41:1012-1021

59. Riley JK, Takeda K, Akira S, Schreiber RD (1999) Interleukin-10 receptor signaling through the JAK-STAT pathway. Requirement for two distinct receptor-derived signals for anti-inflammatory action. J Biol Chem 274:16513-16521

60. Chang J, Kunkel SL, Chang CH (2009) Negative regulation of MyD88-dependent signaling by IL-10 in dendritic cells. Proc Natl Acad Sci USA 106:18327-18332

61. Brint EK et al (2004) ST2 is an inhibitor of interleukin 1 receptor and Toll-like receptor 4 signaling and maintains endotoxin tolerance. Nat Immunol 5:373-379

62. Jovanovic I et al (2011) ST2 deletion enhances innate and acquired immunity to murine mammary carcinoma. Eur J Immunol 41:1902-1912

63. Kobayashi K et al (2002) IRAK-M is a negative regulator of Tolllike receptor signaling. Cell 110:191-202

64. Wald D et al (2003) SIGIRR, a negative regulator of toll-like receptor-interleukin 1 receptor signaling. Nat Immunol 4:920-927

65. Ho JE et al (2013) Common genetic variation at the IL1RL1 locus regulates IL-33/ST2 signaling. J Clin Invest 123:4208-4218

66. Bandara G, Beaven MA, Olivera A, Gilfillan AM, Metcalfe DD (2015) Activated mast cells synthesize and release soluble ST2-a decoy receptor for IL-33. Eur J Immunol 45:3034-3044

67. Sala Frigerio C et al (2019) The major risk factors for Alzheimer's disease: age, sex, and genes modulate the microglia response to $\mathrm{A} \beta$ plaques. Cell Rep 27:1293-1306.e6

68. Keren-Shaul $\mathrm{H}$ et al (2017) A unique microglia type associated with restricting development of Alzheimer's disease. Cell 169:12761290.e17

69. Davalos D et al (2005) ATP mediates rapid microglial response to local brain injury in vivo. Nat Neurosci 8:752-758

70. Madry C et al (2018) Microglial ramification, surveillance, and interleukin-1 $\beta$ release are regulated by the two-pore domain $\mathrm{K}+$ channel THIK-1. Neuron 97:299-312.e6

71. Kim J, Basak JM, Holtzman DM (2009) The role of apolipoprotein E in Alzheimer's disease. Neuron 63:287-303

72. Ulrich JD et al (2018) ApoE facilitates the microglial response to amyloid plaque pathology. J Exp Med 215:1047-1058

73. Liu $S$ et al (2012) TLR2 is a primary receptor for Alzheimer's amyloid $\beta$ peptide to trigger neuroinflammatory activation. J Immunol 188:1098-1107 
74. Krasemann S et al (2017) The TREM2-APOE pathway drives the transcriptional phenotype of dysfunctional microglia in neurodegenerative diseases. Immunity 47:566-581.e9

75. Parhizkar S et al (2019) Loss of TREM2 function increases amyloid seeding but reduces plaque-associated ApoE. Nat Neurosci 22:191-204

76. Jay TR et al (2017) Disease progression-dependent effects of TREM2 deficiency in a mouse model of Alzheimer's disease. J Neurosci 37:637-647

77. Griciuc A et al (2013) Alzheimer's disease risk gene cd33 inhibits microglial uptake of amyloid beta. Neuron 78:631-643
78. Podleśny-Drabiniok A, Marcora E, Goate AM (2020) Microglial phagocytosis: a disease-associated process emerging from Alzheimer's disease genetics. Trends Neurosci 43:965-979

Publisher's Note Springer Nature remains neutral with regard to jurisdictional claims in published maps and institutional affiliations. 